\title{
Liver immune responses to inflammatory stimuli in a diet- induced obesity model of zebrafish
}

\author{
Gabriel Forn-Cuní, Monica Varela, Conrado M Fernández-Rodríguez', \\ Antonio Figueras and Beatriz Novoa
}

Instituto de Investigaciones Marinas, CSIC, Eduardo Cabello 6, 36208 Vigo, Spain

${ }^{1}$ Hospital Universitario Fundación Alcorcón, Madrid, Spain
Correspondence should be addressed to B Novoa

Email

virus@iim.csic.es

\begin{abstract}
Obesity- and metabolic syndrome-related diseases are becoming important medical challenges for the western world. Non-alcoholic fatty liver disease (NAFLD) is a manifestation of these altered conditions in the liver, and inflammation appears to be a factor that is tightly connected to its evolution. In this study, we used a diet-induced obesity approach in zebrafish (Danio rerio) based on overfeeding to analyze liver transcriptomic modulation in the disease and to determine how obesity affects the immune response against an acute inflammatory stimulus such as lipopolysaccharide (LPS). Overfed zebrafish developed an obese phenotype, showed signs of liver steatosis, and its modulation profile resembled that observed in humans, with overexpression of tac4, col4a3, col4a5, lysyl oxidases, and genes involved in retinoid metabolism. In response to LPS, healthy fish exhibited a typical host defense reaction comparable to that which occurs in mammals, whereas there was no significant gene modulation when comparing expression in the liver of LPS-stimulated and non-stimulated obese zebrafish at the same statistical level. The stimulation of obese fish represents a double-hit to the already damaged liver and can help understand the evolution of the disease. Finally, a comparison of the differential gene activation between stimulated healthy and obese zebrafish revealed the expected difference in the metabolic state between healthy and diseased liver. The differentially modulated genes are currently being studied as putative new pathological markers in NAFLD-stimulated liver in humans.
\end{abstract} Key Words - NAFLD - DIO

- inflammation - zebrafish

\section{Introduction}

Obesity is a major risk factor for a number of chronic diseases, including diabetes mellitus, cardiovascular diseases, and cancer, and according to the World Health Organization, it has become a health challenge of epidemic proportions in the last few decades in the western and westernized societies (Bessesen 2008). Increased adiposity causes the deregulation of energetic homeostatic processes, which ultimately manifests as insulin resistance, diabetes, and dyslipidemia. These diseases are part of a condition termed metabolic syndrome. The hepatic component of metabolic syndrome is non-alcoholic fatty liver disease (NAFLD). This term comprises a wide range of progressive liver injuries including bland steatosis, variable degrees of liver inflammation, liver cell necrosis (hepatocyte ballooning), and liver fibrosis, which is also termed non-alcoholic

Published by Bioscientifica Ltd. 
steatohepatitis (NASH). NASH is thought to be the main cause of human cryptogenic cirrhosis. Histologically, NASH is similar to alcoholic liver injury, and the conditions are differentiated by the absence of alcohol abuse in individuals with NASH. NASH is the leading cause of chronic liver disease in both western countries and Asia, and it also increases the risk for hepatocellular carcinoma development (Duan et al. 2013, Yu et al. 2013).

Inflammation is closely connected to the evolution of NAFLD into NASH. In particular, the host inflammatory response to gut microbiota is emerging as a key player in the development and pathogenicity of NASH (Abu-Shanab \& Quigley 2010). Liver injury may result from dysbiosis and bacterial overgrowth, resulting in bacterial translocation and, in turn, contributing to liver injury (Fouts et al. 2012). The increased presence of endogenous bacterial products such as LPS and bacterial DNA activates the host's TLR4 and TLR9 signaling pathways (Henao-Mejia et al. 2013). At the same time, TLR2 activation can be triggered by hepatic lipid accumulation (Miura et al. 2013). Upon activation, TLR2, TLR4, and TLR9 signal through the MyD88 pathway, activate $\mathrm{NF}-\kappa \beta$, and result in the production of proinflammatory cytokines and chronic inflammation (Miura et al. 2013, Roh \& Seki 2013).

The zebrafish model is becoming a valuable tool for improving our understanding of obesity and its associated diseases (Schlegel \& Stainier 2007). Larvae have started to be used in genetic and chemical screenings for the development of drugs targeting obesity (Jones et al. 2008, Chu et al. 2012), and NAFLD and NASH have been reported in various fish models (Asaoka et al. 2013). For example, fgr mutant (Sadler et al. 2005) and transgenic zebrafish expressing the hepatitis B virus X protein (Shieh et al. 2010) display most of the mammalian NASH features. Furthermore, NAFLD can be induced in healthy zebrafish both chemically, with tamoxifen (Anezaki et al. 2009), and with a high-fat content diet (Oka et al. 2010). The diet-induced obesity (DIO) approach allows the modeling of NAFLD in the context of systematic obesity, hence mimicking the most common process occurring in humans suffering this condition. A DIO zebrafish model has already been proven useful to investigate the effects of different compounds in obesity and, specifically, in the steatotic liver (Tainaka et al. 2011). In this study, we used a DIO zebrafish model based on overfeeding to analyze the modulation of the liver transcriptomic response and to examine how obesity and, in particular, NAFLD affect the immune response against a powerful inflammatory stimulus such as lipopolysaccharide (LPS).

\section{Materials and methods}

\section{Animals}

WT zebrafish were obtained from our experimental facilities where zebrafish were cultured following established protocols (Westerfield 2000, Nusslein-Volhard \& Dahm 2002) (also see http://zfin.org/zf_info/zfbook/zfbk. html). Fish care and challenge experiments were conducted according the CSIC National Committee on Bioethics guidelines under approval number ID 01_09032012. Control fish were fed two times a day, once with Artemia and once with standard fish chow (Nutrafin Max, Hagen, Germany), while obese fish were fed fish chow two more times a day (Fig. 1A). Typical fish chow primarily consisted of $46.5 \%$ protein, $11.5 \%$ oils and fat, $8.5 \%$ ash, and $1.4 \%$ fiber. After 8 months of the differential diet, PBS or $10 \mu \mathrm{g}$ LPS (Sigma L2630) was intraperitoneally injected into control and obese fish. For every treatment, four biological replicates including the liver from three fish were pooled $3 \mathrm{~h}$ post-injection and stored at $-80^{\circ} \mathrm{C}$ until use for microarray analysis. Furthermore, kidney and muscle were also collected at the same times and their $i l 1 b$ and il6 expression analyzed by real-time PCR in order to confirm a systemic inflammation in response to LPS (Supplementary Figure 1A, see section on supplementary data given at the end of this article).

Additional fish were also sampled and liver samples were taken for histological analysis following standard procedures: frozen zebrafish liver samples were used for Oil-Red O staining following Mehlem et al. (2013) and fixed liver samples were embedded in paraffin and stained with hematoxylin and eosin (Merck). Histological sections were examined under light microscopy (Nikon Eclipse 80i).

\section{RNA isolation and cDNA transcription}

RNA was extracted using TRIzol reagent (Life Technologies) following the TRIzol manufacturer's specifications in combination with the RNeasy Mini Kit (Qiagen); the extracted RNA was preserved at $-80^{\circ} \mathrm{C}$ until use. After DNase I treatment, $1 \mu \mathrm{g}$ total RNA was used to obtain cDNA using the SuperScript III First-Strand Synthesis SuperMix for quantitative RT-PCR (qRT-PCR) (Life Technologies).

\section{Microarray analyses}

The $4 \times 44 \mathrm{~K}$ Zebrafish Gene Expression Microarray (V3, AMADID 026437) containing 43803 probes representing

Published by Bioscientifica Ltd. 
A
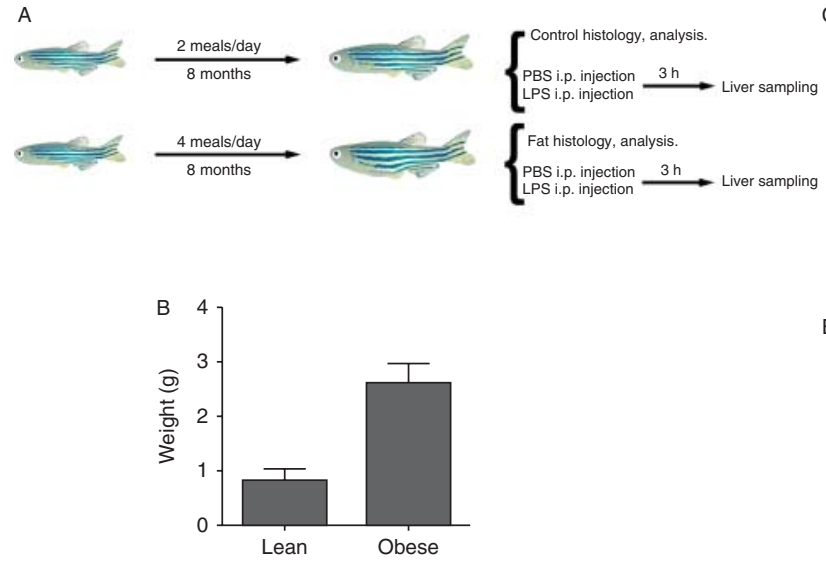
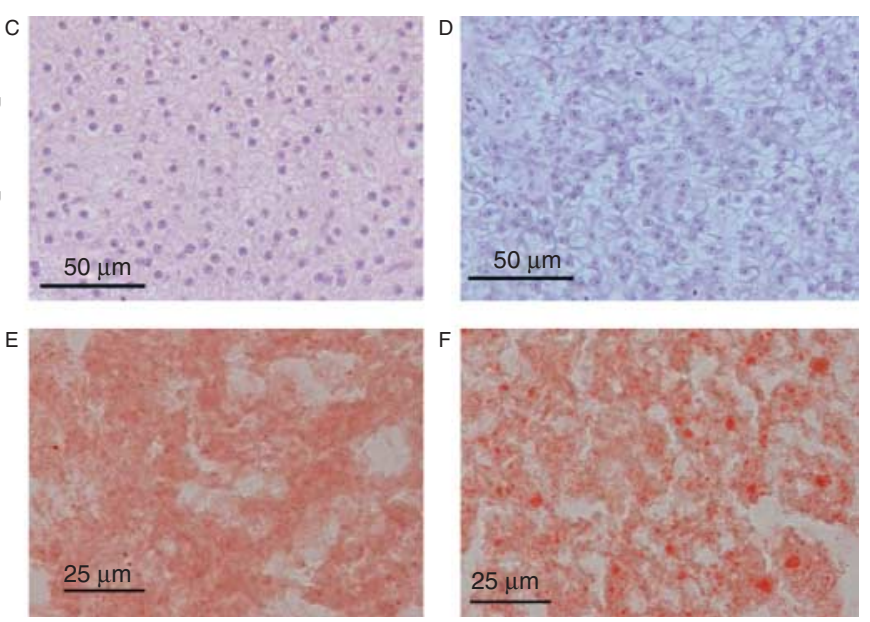

Figure 1

(A) Simple diagram of the experiment. (B) Weight difference between control and overfed zebrafish. (C) Histological samples of healthy zebrafish liver and (D) obese zebrafish liver. The liver of overfed zebrafish present

23207 genes was used (Agilent Technologies, Madrid, Spain). RNA quality was assessed using the Agilent 2100 Bioanalyzer and stored frozen at $-80^{\circ} \mathrm{C}$ until all the RNA could be hybridized and processed simultaneously. The labeling of $2 \mu \mathrm{g}$ RNA $(\sim 50 \mu \mathrm{g} / \mathrm{ml})$ and hybridization were carried out using the Universidad Autónoma de Barcelona microarray platform, complying with the minimum Information about a microarray experiment standards (Brazma et al. 2001). The signal was captured, processed, and segmented using an Agilent G2565B scanner (Agilent Technologies) with Agilent Feature Extraction Software (v9.5) protocol GE1-v5_95 using an extended dynamic range and preprocessing by Agilent Feature Extraction v9.5.5.1.

The results for the fluorescence intensity data and quality annotations were imported into GeneSpring GX version 12.6 (Agilent Technologies). All the control features (including the positive and negative controls and the landing lights) were excluded from subsequent analyses. Normalization was then carried out by a percentile shift at the 75th percentile. Entities with an expression between the 20th and 95th percentiles in the raw data were retained and used in subsequent analyses. To assess genes for differential expression, the normalized $\log$ intensity ratios were analyzed with a moderated $T$-test with Benjamini-Hochberg FDR multiple testing correction, and significance was established at a corrected $P<0.1$. A reannotation of the microarray was performed using the most recent information for Danio rerio sequences on the Ensembl genome and NCBI nucleotide RefSeq databases. symptoms of steatosis such as cell vacuolization. (E) Oil-Red O lipid staining of healthy and (F) overfed zebrafish.

The microarray expression values were validated with quantitative PCR (qPCR) analysis of the expression of six different genes in the RNA samples used to hybridize the array. qPCR and microarray fold-changes were correlated using Spearman rank correlation analysis (Supplementary Figure 2, see section on supplementary data given at the end of this article).

The values obtained using microarray were compared with other already published models using gene set enrichment analysis (GSEA) with default parameters (Subramanian et al. 2005). Obesity-related datasets were retrieved from published studies: zebrafish DIO (GSE18566) (Oka et al. 2010), rat DIO (GSE8700) (Li et al. 2008), mice DIO (GSE11790) (Poussin et al. 2008), and human adipocytes in obesity (GSE15524 and GSE2952) (Nadler et al. 2000, MacLaren et al. 2010). For the LPS comparison, the curated group of datasets C7 (immunological signatures) of the MSigDB was used.

Visualization of the Gene Ontology biological function terms network in the overfed zebrafish liver was performed using Cytoscape v3.0.2 with the ClueGO plugin (Bindea et al. 2009). For enrichment analysis, identification of functional classes that significantly differed among treatments was carried out using both the Blast2GO suite (Conesa et al. 2005) and the DAVID online platform (Huang et al. 2009a,b), selecting the Agilent Microarray as the background and default options. Visualization of the KEGG categories in the different treatments was performed with Circos (Krzywinski et al. 2009).

Published by Bioscientifica Ltd. 


\section{Quantitative RT-PCR}

Specific PCR primers were designed from the sequences of the selected probes (Supplementary Table 1, see section on supplementary data given at the end of this article) using the Primer3 program (Rozen \& Skaletsky 2000) according to qRT-PCR restrictions. Oligo Analyzer 1.0.2 was used to check for dimer and hairpin formation. The efficiency of each primer pair was then analyzed with seven serial fivefold dilutions of cDNA of zebrafish and calculated from the slope of the regression line of the quantification cycle vs the relative concentration of cDNA (Pfaffl 2001). A melting curve analysis was also performed to verify that only specific amplification occurred and that no primer dimers were amplified. If these conditions were not met, new primer pairs were designed.

qRT-PCR was performed using a 7300 real time PCR system (Applied Biosystems). One microliter of fivefold diluted cDNA template was mixed with $0.5 \mu \mathrm{l}$ of each primer $(10 \mu \mathrm{M})$ and $12.5 \mu$ l of SYBR green PCR master mix (Applied Biosystems) in a final volume of $25 \mu \mathrm{l}$. The standard cycling conditions were $95^{\circ} \mathrm{C}$ for $10 \mathrm{~min}$, followed by 40 cycles of $95^{\circ} \mathrm{C}$ for $15 \mathrm{~s}$ and $60{ }^{\circ} \mathrm{C}$ for $1 \mathrm{~min}$. All reactions were performed as technical triplicates, and an analysis of melting curves was performed in each reaction. The relative expression levels of the genes were normalized using 18S ribosomal RNA (BX296557) expression as a housekeeping gene control, which was constitutively expressed and not affected by the treatments, following the Pfaffl (2001) method. Fold-change units were calculated by dividing the normalized expression values of stimulated tissues by the normalized expression values of the controls. For the biological replicates, the average relative level of expression from each replicate was considered as a single point, and the mean and s.e. were calculated.

\section{Results}

\section{The DIO zebrafish model}

After 8 months of a differential diet (Fig. 1A), the differences between normal (animals fed with standard diet) and overfed zebrafish were apparent. Control animals were fit and weighed an average of $0.8 \mathrm{~g}$, while overfed zebrafish were obese and weighed an average of $2.1 \mathrm{~g}$, which was 2.6-fold greater than the control fish (Fig. 1B). In fact, an increase of $2.26 \pm 0.37 \mathrm{~g}$ was observed during the DIO experiments (data not shown). NAFLD was evaluated by histological analysis of liver samples. Liver tissue from zebrafish fed the standard diet appeared healthy (Fig. 1C). Signs of steatosis were detected in the liver of overfed individuals (Fig. 1D). Moreover, lipid staining revealed lipid accumulations in overfed zebrafish while none was found in healthy fish (Fig. 1E and F).

\section{The liver gene expression profile of obese fish}

The liver transcriptomic analysis in this study was approached using microarrays, and all the data and results can be found on the Gene Expression Omnibus (GEO) under accession number GSE56478. Overfeeding significantly affected the expression of 196 annotated genes; 177 were overexpressed while only 17 were inhibited (Fig. 2). Together with the rest of the comparisons in this study, the fold-change of all the statistically significant genes in this analysis can be found in Supplementary Table 2, see section on supplementary data given at the end of this article. GSEA was used to highlight the similarities between the DIO-modulated genes in the obese zebrafish and other DIO models and obesity datasets (Supplementary Table 3A). We performed a first-approach analysis by mapping the regulated genes to their significant Gene Ontology terms. The obtained gene expression profile resembled that observed in obese humans with NAFLD, with predicted biological functions

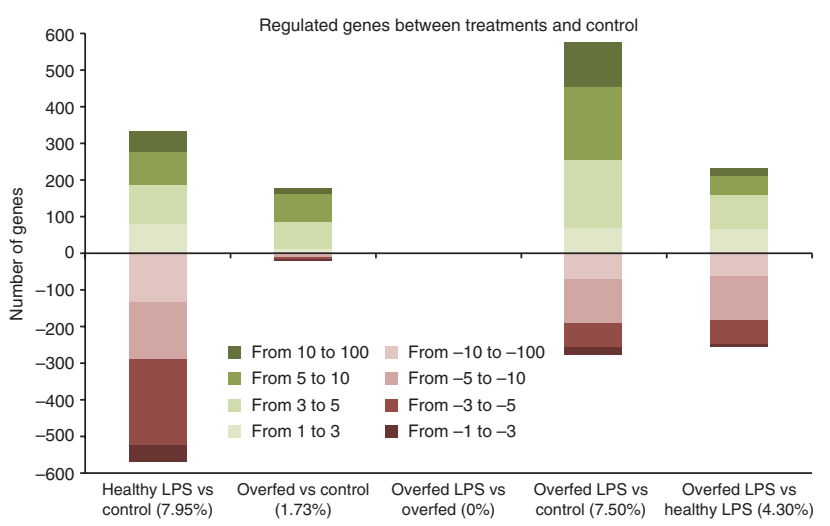

\section{Figure 2}

The number of genes and fold-change between the different treatments analyzed in this study. The percentage of well-annotated modulated genes relative to the total present in at least two treatment comparisons is indicated in parentheses. In control zebrafish, more than 300 genes were overexpressed in response to LPS, while more than 500 were inhibited. Obesity alone modulated 195 genes of differential pathways related to NAFLD. No significant gene modulation was reported $3 \mathrm{~h}$ after LPS was intraperitoneally injected into obese zebrafish. However, 850 genes, most of them upregulated, were significantly altered in the obese stimulated liver with respect to the control. 


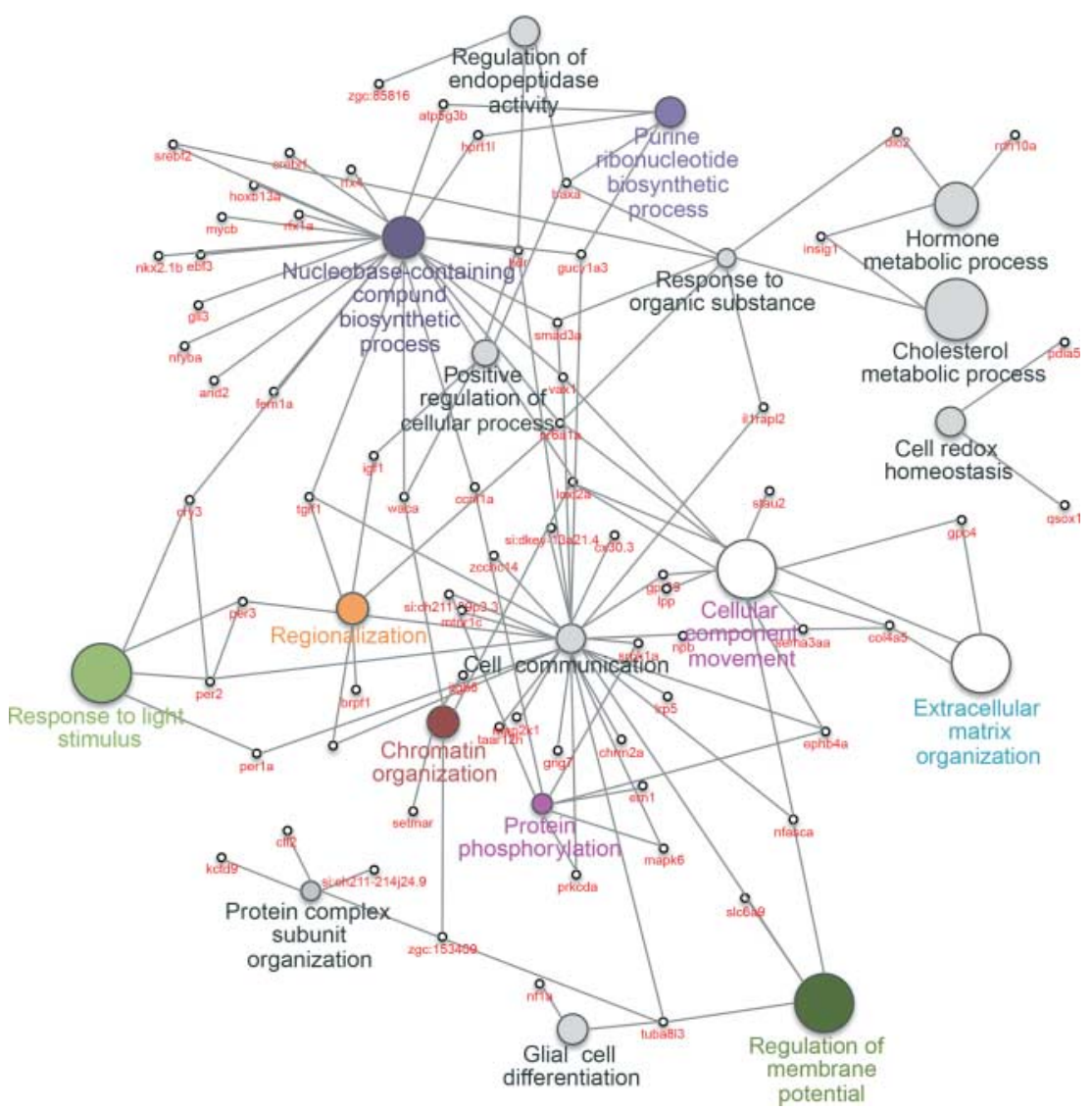

\section{Figure 3}

Visualization of the most significant biological function-categorized Gene Ontology terms related to the regulated genes in the obese zebrafish.

such as extracellular matrix organization, response to light stimulus, and effects on hormone metabolic processes (Fig. 3). NAFLD is a multifactorial condition that affects several cellular pathways. In consequence, most of the regulated genes affected by the diet reflected this variety of NAFLD-responding routes (Table 1): immune response and GTPases (gimap7, gimap8l, and ifi44), energy metabolism and oxidative phosphorylation (atp5e and atp5g3b), lipid metabolism (fam57b), protein folding and ubiquitination (crebrf, ugt5a2, and uba52), neuronal-related genes (tac4a, mpzl2, cbln4, vax1, znf512b, and tuba1al), modification of the extracellular matrix (col4a2, col4a5, and loxl2a), and retinoid metabolism (rbp2). Interestingly, enrichment analysis predicted that the circadian rhythm was the only statistically enriched KEGG pathway in the pathological liver from obese individuals compared with controls (corrected $P$ value $=0.00076$ ). Belonging to this pathway, Period family genes per1a, per2, and per 3 and cry 3 genes were overexpressed by 7.3-, 2.7-, 4.1-, and 2.4-fold respectively in obese zebrafish. Printed in Great Britain
The size of the Gene Ontology term is relative to its $P$ value in an inverse correlation.

Differential immune response against an inflammatory stimulus in normal and overfed fish

A total of 902 genes demonstrated significantly altered expression $3 \mathrm{~h}$ after a sublethal LPS inoculation in healthy fish (Fig. 2). The analysis of these LPS-regulated genes in zebrafish with the C7 database of immunological signatures using GSEA confirmed the enrichment of the genes and pathways involved in this response on mammals and other organisms (Supplementary Table 3B, see section on supplementary data given at the end of this article). The significantly enriched KEGG pathways found in the LPS response were related to antigen detection and processing (Toll-like receptor signaling, RIG-I-like receptor signaling, NOD-like receptor signaling, cytosolic DNA-sensing pathway, and ubiquitin-mediated proteolysis), immune signaling and cytokine release (Jak-STAT signaling, MAPK signaling pathway, and cytokine-cytokine receptor interaction), and cell cycle regulation (cell cycle, p53 signaling, and apoptosis) (Table 2). The enriched Gene

Published by Bioscientifica Ltd 
Table 1 Most regulated genes between the liver of obese and control zebrafish

\begin{tabular}{lll}
\hline Fold-change & & NCBI gene ID \\
\cline { 1 - 2 } 119.37 & 503941 \\
29.42 & 798291 \\
29.24 & 100151267 \\
29.16 & 767756 \\
28.91 & 100150568 \\
19.29 & 100535622 \\
17.76 & 100002814 \\
16 & 81541 \\
15.97 & 100534682 \\
13.68 & 100038773 \\
13.61 & 795887 \\
13.36 & 799650 \\
12.71 & 100006971 \\
12.4 & 563847 \\
11.12 & 565508 \\
10.46 & 373870 \\
-10.41 & 64886 \\
10.23 & 100141490 \\
9.97 & 101885988 \\
9.87 & 100149876 \\
9.72 & 795591 \\
9.66 & 641289 \\
9.6 & 793072 \\
9.57 & 432384 \\
9.48 & 100003412 \\
\hline & \\
\hline
\end{tabular}

Gene symbol
gimap7
atp5e
tac4
ugt5a2
rnf11b
loc100535622
fam57b
atp5g3b
mpzl2
rfx1a
ifi44
ddx21
trim59l
cbln4
loxl2a
vax1
mhc1ufa
nfasca
znf512b
crebrf
tuba1al
uba52
gimap8l
rbp2b
zgc:172139

\section{Gene name}

GTPase, IMAP family member 7

ATP synthase, $\mathrm{H}+$ transporting, mitochondrial F1 complex, epsilon subunit Tachykinin 4 (hemokinin)

UDP glucuronosyltransferase 5 family, polypeptide A2

Ring finger protein $11 \mathrm{~b}$

Uncharacterized LOC100535622

Family with sequence similarity 57 , member B

ATP synthase, $\mathrm{H}+$ transporting, mitochondrial F0 complex, subunit C3

Myelin protein zero-like protein 2-like

Regulatory factor $\mathrm{X}, 1$ a (influences HLA class II expression)

Interferon-induced protein 44

DEAD (asp-glu-ala-asp) box polypeptide 21

Tripartite motif-containing protein 59-like

Cerebellin 4 precursor

Lysyl oxidase-like 2a; two homologs

Ventral anterior homeobox 1

Major histocompatibility complex class I UFA

Neurofascin homolog (chicken) A

Zinc finger protein 512B

CREB3 regulatory factor

Tubulin $\alpha$-1A chain-like

Ubiquitin A-52 residue ribosomal protein fusion product 1

GTPase IMAP family member 8-like

Retinol binding protein $2 \mathrm{~b}$, cellular

Uncharacterized KIAA0930-like

Ontology terms of the stimulated samples were in concordance and were primarily associated with the immunological response to a bacterial stimulus, cytokine signaling, and the regulation of apoptosis. Although the majority of the gene regulation following LPS treatment was inhibitory, the genes with the greatest alterations were primarily upregulated, with $n r O b 2 b$ being the most overexpressed; $n r O b 2 b$ is an orphan receptor with several liver-related functions, and it demonstrated a fold-change of 85.76. As expected, PAMP-recognition molecules (tlr5b), pro-inflammatory cytokines (il1b and il8), and chemokines (cxcl-c1c and $c x c l 11 l)$, acute phase proteins (saa), and cell cycle regulation (diabloa) genes were some of the most modulated genes in response to LPS.

To determine how the liver of obese zebrafish responded to the inflammatory stimulus, we also injected LPS intraperitoneally into obese zebrafish using the same method as normal fish. Surprisingly, at the same significance level used for the rest of the experiment, no genes were modulated when the liver gene expression profile of LPS-stimulated obese zebrafish was compared with that of non-stimulated obese zebrafish. This contrasts with the strong and defined response to LPS in healthy fish and suggests that obese zebrafish have an impaired immune response (Fig. 2).

We also compared the expression of the stimulated obese zebrafish to the non-stimulated controls. In this scenario, the expression of 850 genes was significantly altered, which was increased from the 196 genes with altered expression induced by obesity alone. Moreover, $134(68 \%)$ of the regulated genes in the obese zebrafish were also modulated in the LPS response, and $66.5 \%$ of them had the same regulation directionality. As in healthy fish, analysis of enriched pathways in the obese response to LPS appeared immune associated (Jak-STAT signaling pathway, NOD-like receptor signaling pathway, Toll-like receptor signaling pathway, MAPK signaling pathway, cytokine-cytokine receptor interaction and apoptosis) (Table 2).

The different metabolic states in the healthy and diseased liver were clearly observed in the direct comparison between the healthy and obese LPS-stimulated zebrafish. The PPAR signaling pathway, retinoic and sphingolipid metabolism were significantly altered in the stimulated overfed zebrafish when compared with stimulated lean fish (Table 2). Interestingly, some immune genes with primary liver expression were found to have more greatly altered expression in the obese zebrafish liver (Supplementary Figure 1B). For example, the two known hepcidin orthologs in zebrafish, hamp1 and hamp2, were upregulated by 14-fold in the inflammatory process in the NAFLD liver but were not altered in the healthy liver. Similarly, the saa gene was one of the most differentially

Published by Bioscientifica Ltd 
Table 2 Differential KEGG pathway enrichment in the LPS response between healthy and obese zebrafish

Term

(A) Enriched KEGG pathways in the healthy zebrafish liver LPS response Toll-like receptor signaling pathway

Cell cycle

Jak-STAT signaling pathway

Apoptosis

RIG-I-like receptor signaling pathway

Oocyte meiosis

Ubiquitin mediated proteolysis

MAPK signaling pathway

p53 signaling pathway

Cytokine-cytokine receptor interaction

NOD-like receptor signaling pathway

Progesterone-mediated oocyte maturation

Cytosolic DNA-sensing pathway

(B) Enriched KEGG pathways in the obese zebrafish liver LPS response Apoptosis

Jak-STAT signaling pathway

Toll-like receptor signaling pathway

MAPK signaling pathway

NOD-like receptor signaling pathway

Natural killer cell mediated cytotoxicity

Cytokine-cytokine receptor interaction

(C) Enriched KEGG pathways in the differential LPS response between healthy and obese zebrafish

Retinol metabolism

PPAR signaling pathway

Sphingolipid metabolism

\begin{tabular}{|c|c|c|c|}
\hline Count & $\%$ & $P$ value & Benjamini \\
\hline 14 & 1.7 & 0.00000084 & 0.00006 \\
\hline 16 & 1.9 & 0.00003 & 0.0011 \\
\hline 11 & 1.3 & 0.0002 & 0.0047 \\
\hline 10 & 1.2 & 0.0013 & 0.023 \\
\hline 7 & 0.8 & 0.0015 & 0.021 \\
\hline 11 & 1.3 & 0.0024 & 0.028 \\
\hline 11 & 1.3 & 0.0038 & 0.039 \\
\hline 16 & 1.9 & 0.0074 & 0.065 \\
\hline 8 & 1 & 0.0079 & 0.061 \\
\hline 6 & 0.7 & 0.034 & 0.22 \\
\hline 5 & 0.6 & 0.052 & 0.3 \\
\hline 7 & 0.8 & 0.065 & 0.33 \\
\hline 4 & 0.5 & 0.093 & 0.42 \\
\hline 10 & 1.3 & 0.00044 & 0.038 \\
\hline 8 & 1 & 0.0066 & 0.26 \\
\hline 8 & 1 & 0.0066 & 0.26 \\
\hline 13 & 1.7 & 0.028 & 0.57 \\
\hline 5 & 0.6 & 0.033 & 0.52 \\
\hline 6 & 0.8 & 0.05 & 0.6 \\
\hline 5 & 0.6 & 0.071 & 0.67 \\
\hline 3 & 0.7 & 0.063 & 0.99 \\
\hline 4 & 0.9 & 0.07 & 0.93 \\
\hline 3 & 0.7 & 0.095 & 0.91 \\
\hline
\end{tabular}

expressed genes in the obese fish response to LPS and was overexpressed up to 3.88-fold in the stimulated obese liver when compared with the stimulated healthy liver. Moreover, ifi44 was one of the most overexpressed genes in the liver of overfed zebrafish after LPS stimulation, which was similar to the observations in non-stimulated obese zebrafish and in contrast to the low modulation in healthy individuals.

Finally, upon a more in-depth analysis, obese zebrafish appeared unable to modify the expression of a great number of genes that take part in a healthy inflammatory process (Fig. 4A and B). The stimulation of normal animals affected the regulation of immune system genes in the liver, including those belonging to the Toll-like receptor signaling pathway, ubiquitin-mediated proteolysis, the RIG-I-like receptor signaling pathway, the MAPK pathway, and the Jak-STAT signaling pathway, which were not altered in the overfed zebrafish. Furthermore, the apoptotic balance appeared differently regulated between the healthy and obese inflammatory response (Supplementary Figure 1C). The expression of both pro-apoptotic (casp7, casp8, casp9, birc2, and cflara) and anti-apoptotic genes (diabloa, birc2, and bric5b) was modulated in the healthy inflammatory response. In the NAFLD fish model, however, stimulation with LPS primarily activated anti-apoptotic and proto-oncogenes (card9, birc2, bag3, junba, and jun), while it downregulated pro-apoptotic players $(d f f b, b a d b$, and casp $3 a)$ and altered the cellular balance toward cell survival and proliferation after LPS stimulation.

\section{Discussion}

\section{Model characterization}

In this study, we characterized the transcriptomic modulation of a DIO zebrafish model that exhibits the specific signs of the human NAFLD condition, as represented by both differential histological and described gene expression features. The liver of overfed zebrafish mimicked the histological signs of human NAFLD, and the gene modulation demonstrated using microarrays delineated the alterations occurring in the human disease. A first-approach analysis was made by mapping the modulated genes in the liver of obese zebrafish to their Gene Ontology functions and revealed that processes related to the extracellular matrix and metabolism were affected.

Furthermore, the genes with higher fold-changes in the overfed liver belonged to processes known to be

Published by Bioscientifica Ltd. 


\section{A}

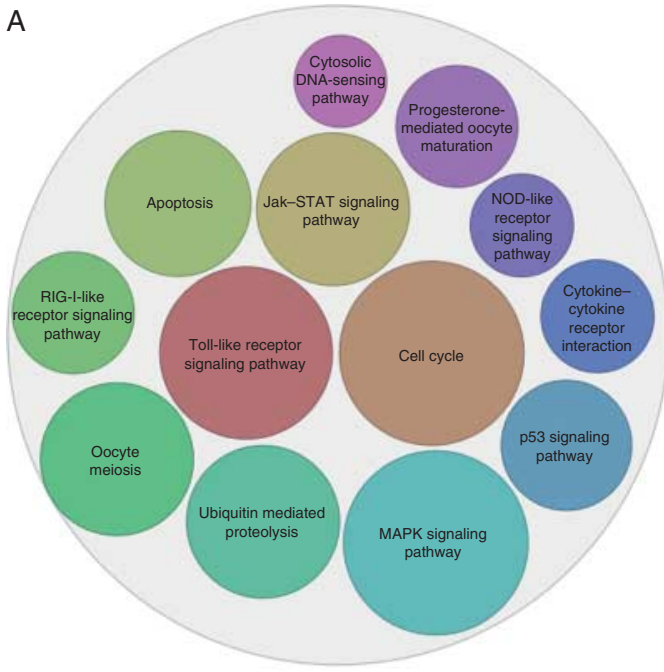

B
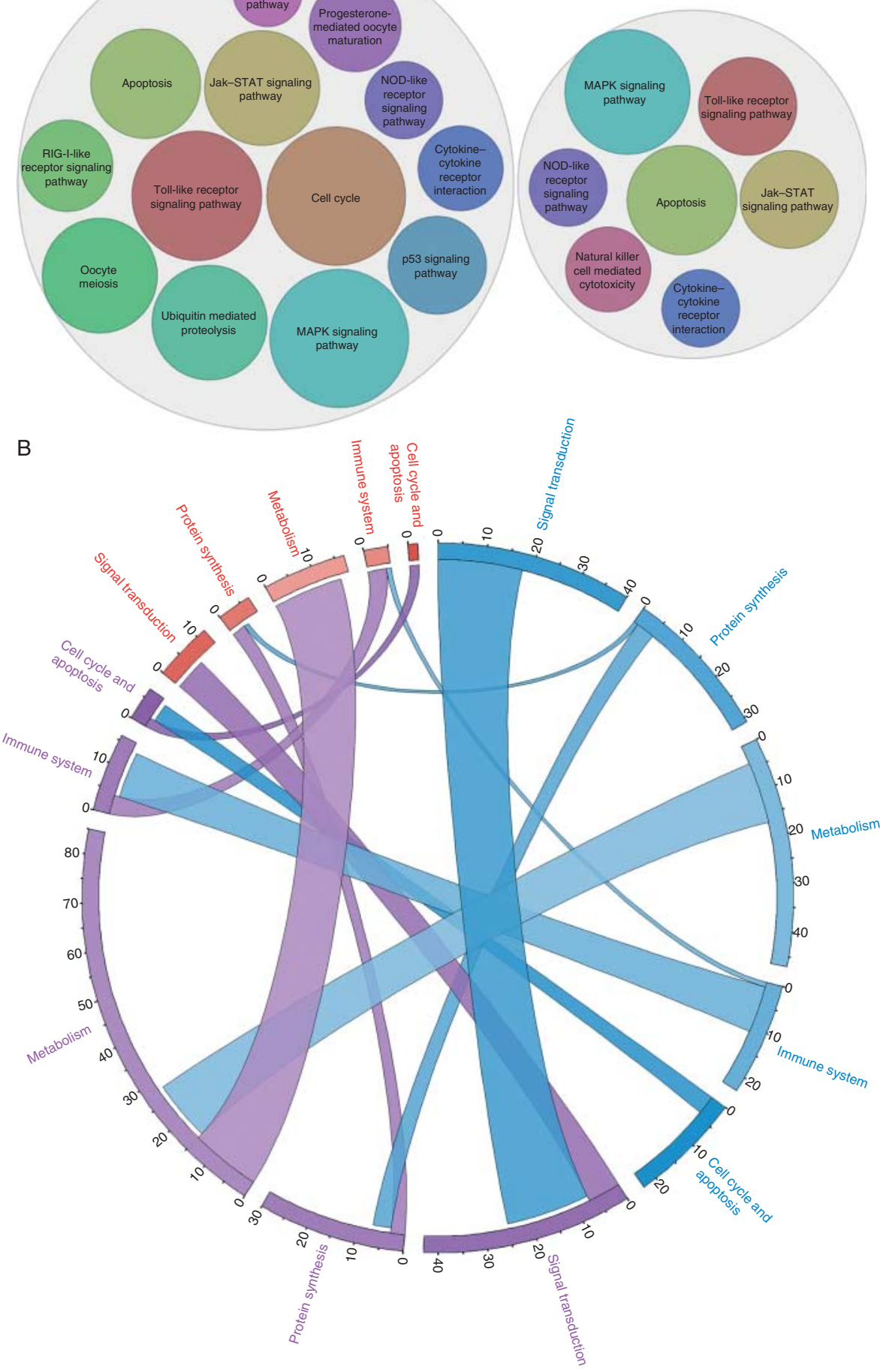

Figure 4

(A) KEGG pathways that were statistically modulated in the healthy fish response to LPS with respect to controls (left), and the obese LPS-stimulated fish with respect to the controls (right). (B) Gene expression correlation between the significantly regulated genes in the different treatments and the healthy controls in different KEGG categories. The ribbons indicate the same genes regulated between the categories in the non-stimulated obese (red), stimulated obese (violet), and stimulated healthy (blue) liver. http://joe.endocrinology-journals.org DOI: 10.1530/JOE-14-0398
(C) 2015 Society for Endocrinology Printed in Great Britain 
affected in the disease. atp5e and atp5gb3b take part in oxidative phosphorylation and energy metabolism, a commonly impaired process in the insulin resistance state that can lead to NASH (Pessayre 2007). fam57b is a $P P A R-\gamma$ target that regulates ceramide biosynthesis, manifesting as regulation of lipid metabolism in obese zebrafish (Yamashita-Sugahara et al. 2013). NAFLD is also known to affect protein folding. One of the most modulated genes in the zebrafish NAFLD liver is crebrf. This gene acts as a negative regulator of the unfolded protein response, which is hyperactivated in this condition (Cinaroglu et al. 2011). Furthermore, ubiquitination was also altered through the modulation of ugt $5 a 3$ and uba52. Another of the most regulated genes was tac $4 a$, which encodes a family of species-divergent neurotransmitters called tachykinins (Page 2004, 2006). Although the exact function of tac4 paralogs in zebrafish has not been unveiled, hemokinin 1 (Hk1), a product of the Tac4 gene, is reported to be expressed along with substance $\mathrm{P}$ at sites of chronic inflammation in mice (Metwali et al. 2004), a typical NAFLD feature. It is well documented that the chronic inflammation caused by metabolic syndrome is one of the main causes of NAFLD evolution into $\mathrm{NASH}$, which can eventually cause liver fibrosis, a process characterized by the deposition of new collagen and matrix molecules in the extracellular space. In particular, typical NAFLD/NASH fibrosis forms a 'chicken-wire' pattern, primarily composed of type IV collagen (Armutcu et al. 2013). In our microarray analysis, col4a2 and col4a5 were found to be significantly upregulated in the liver of overfed zebrafish. Furthermore, loxl2a expression, one of the two loxl2 homologs in zebrafish, was 11-fold higher in overfed fish. Lysyl oxidases are proteins that catalyze the polymerization of collagen, and LOXL2 gene expression has been previously associated with liver fibrosis (Vadasz et al. 2005). Moreover, $r b p 2$, also modulated in the obese zebrafish, is involved in retinoid metabolism. Retinoids are anti-inflammatory molecules that promote cellular differentiation. The liver is the most important site for retinoid storage, and its deregulation is associated with hepatic fibrosis and hepatocarcinoma (Shirakami et al. 2012). Interestingly, an important group of these most regulated genes in the obese liver are primarily characterized for their neuronal related function, including mpzl2, cbln4, vax1, znf512b, and tuba1al. Finally, genes putatively related to the immune response such as ifi44 were overexpressed in the liver of overfed zebrafish. Ifi44 has also been markedly induced in the liver in response to hepatitis $\mathrm{C}$ in chimpanzees (Kitamura et al. 1994), although no modulation of this gene had previously been reported in NAFLD. Additionally, two of the most regulated genes, gimap7 and gimap8l, are GTPases of the immunity-associated protein family, which is an evolutionarily conserved group that appears to be immunity modulated (Krücken et al. 2004). However, more studies are needed to decipher the different functions of its members.

Using a pathway-specific analysis, we found that the disease particularly affected the circadian rhythm. The relationship between circadian clocks and metabolism has become an area of focus in the last few years, and the mechanisms by which molecular clocks influence metabolic regulation are being progressively elucidated (Sahar \& Sassone-Corsi 2012). The liver is considered a peripheral clock, in the sense that it can regulate various metabolic pathways depending on the expression of certain molecular clocks such as the PER and CRY family proteins (Sahar \& Sassone-Corsi 2012). There is growing evidence of the reverse process, by which the metabolic state regulates PER2, which in turn controls hepatic glycogen storage/ degradation (Tahara et al. 2011, Zani et al. 2013). Supporting previous findings, per 2 regulation may limit glucose production by increasing glycogen storage or preventing glycogenolysis in obese zebrafish.

\section{Differential immune response against an inflammatory stimulus in normal and overfed fish}

In mammals, microbial LPS is a potent inflammatory inducer that is primarily recognized by TLR4. The intracellular signaling pathway can proceed through either a MyD88-dependent or MyD88-independent manner, ultimately causing the release of pro-inflammatory cytokines. Zebrafish appear to recognize endotoxin in a TLR4-independent manner (Sepulcre et al. 2009, Sullivan et al. 2009), and LPS triggers an inflammatory response powerful enough to induce death (Novoa et al. 2009). As demonstrated in the study, the zebrafish transcriptomic response to LPS in the liver presented a typical host immunological defensive reaction comparable to that occurring in stimulated mammals, modulating genes associated with the detection, processing and signaling of external antigens, and activating immune defense pathways. In particular, $n r 02 b 2 b$, a zebrafish ortholog for nrob2, also known as $S H P$, demonstrated the most altered expression after LPS injection. This gene is an orphan nuclear receptor and functions as a key regulator of liver function, affecting the lipid metabolism, reproductive biology, and energy pathways (Garruti et al. 2012) that appear to be involved in different liver pathologies (Smalling et al. 2013).

Published by Bioscientifica Ltd. 
The LPS injection of obese fish represented a double hit to the already damaged liver. Shockingly, we were unable to identify any statistically significant differences in gene modulation between the stimulated and nonstimulated NAFLD liver. The healthy zebrafish response to LPS is very critical and powerful, and although modulation must occur in the damaged liver, its ability to undergo a powerful functional acute inflammatory response was certainly impaired. However, comparing the stimulated obese zebrafish liver transcriptome with that of the non-stimulated healthy liver may aid our understanding of the synergies and interactions between the disease and inflammation.

At first glance, the differential response between the stimulated obese liver and the healthy liver resembled the same stimulation in the healthy zebrafish, modulating the principal defensive immunological response routes and expressing typical proinflammatory markers. Interestingly, the inflammatory response in overfed zebrafish did not appear to be as functional as that in healthy animals, as the obese zebrafish were unable to modulate the expression of a great number of genes that take part in a healthy inflammatory process, particularly those directly involved in the immune system and the cell cycle and apoptosis. Some genes belonging to inflammatory pathways such as the Toll-like receptor signaling pathway were exclusively modulated in the healthy liver but not in the NAFLD liver. Furthermore, LPS signaling stimulates both pro-apoptotic and anti-apoptotic genes in a regulated balance and directly affects the p53 apoptotic pathway (Tsolmongyn et al. 2013). Although more studies are needed, in NAFLD liver stimulation with LPS activated a greater number of anti-apoptotic and proto-oncogenes, thus weighting the cellular balance toward cell survival and proliferation.

The differential metabolic state was also represented after LPS stimulation, as shown by the differential expression of genes involved in the PPAR signaling pathway, retinoic, and sphingolipid metabolism in the healthy stimulated and obese stimulated liver. The PPAR signaling pathway is known to be involved in chronic inflammation and the cirrhotic process of obesity-induced NAFLD (Radonjic et al. 2009). Moreover, retinoids are anti-inflammatory molecules that promote cellular differentiation. The liver is the most important site for retinoid storage, and deregulation of this storage is associated with hepatic fibrosis and hepatocarcinoma (Shirakami et al. 2012).

At a more detailed level, the most regulated genes between both the stimulated obese and healthy zebrafish liver were related to the carcinogenic process. mhcluba is one of the class I histocompatibility antigens in zebrafish (Michalová et al. 2000). Although it may be the consequence of different haplotypes in the WT zebrafish population, the regulation of MHC I genes is found in a great number of cancers. arhgap28, the most overexpressed gene between the obese stimulated and healthy stimulated fish, is a signal transducer that is upregulated in grade II and III meningiomas (Fèvre-Montange et al. 2009). Moreover, hepcidins and saa genes also exhibited different expression profiles following the stimulation of obese and healthy zebrafish. Hepcidins are antimicrobial peptides that are also associated with liver iron metabolism (for example, in iron recycling), and thus, increased hepcidin expression may indicate hepatocyte dysfunction. Furthermore, NAFLD with iron overload increases hepatic damage and is associated with increased levels of hepcidin (Aigner et al. 2008). In addition, hepatic iron overload results in greater liver damage in human NAFLD (Kowdley et al. 2012). Similarly, the saa gene was one of the most differentially expressed genes in the obese fish response to LPS; this is consistent with previous findings, as it has been proposed as a biomarker for insulin resistance (Scheja et al. 2008) and its expression is elevated during hepatic inflammation in mice on a methionine-choline-deficient diet, which is a common rodent model for NASH (Pelz et al. 2012).

In conclusion, we developed an obesity model in zebrafish based on overfeeding that is consistent with previous NAFLD models and the human disease itself. We used this model to study how metabolic syndrome modulates the immune response in the diseased liver. Our results indicate that zebrafish suffering from obesity-induced NAFLD exhibit slightly greater expression of pro-inflammatory markers but are not able to activate all the pathways of a complete inflammatory response. This finding is most likely due to the ongoing chronic inflammation process driven by metabolic factors. Furthermore, we found that after a strong inflammatory stimulus, the apoptotic balance in obese zebrafish was severely weighted toward cell proliferation, while there was equilibrium between proand anti-apoptotic agents in the healthy response. These differences regarding how the diseased liver responds to acute inflammation may aid our understanding of the association between NAFLD and the increased incidence of hepatocarcinoma. We hope that this model can shed some light on the processes occurring in this disease.

\section{Supplementary data}

This is linked to the online version of the paper at http://dx.doi.org/10.1530/ JOE-14-0398.

Published by Bioscientifica Ltd. 


\section{Declaration of interest}

The authors declare that there is no conflict of interest that could be perceived as prejudicing the impartiality of the research reported.

\section{Funding}

This work was supported by the projects CSD2007-00002 'AQUAGENOMICS' of the program Consolider-Ingenio 2010, Spanish Ministerio de Ciencia e Innovación (MICINN); 289209 'FISHFORPHARMA' (EU); and 201230E057 'Proyecto Intramural Especial, PIE', Agencia Estatal Consejo Superior de Investigaciones Científicas (CSIC). M V received a predoctoral grant from the JAE Program (CSIC and European structural Funds).

\section{Author contribution statement}

A F, C M F-R, and B N designed the work; G F-C and $M V$ performed the experiments. A F, C M F-R, B N, G F-C, and M V analyzed and interpreted data. G F-C, A F, C M F-R, and B N wrote the manuscript.

\section{Acknowledgements}

We would like to thank Ruben Chamorro for his technical assistance. Also, we would like to thank the 'Ecobiomar' group at the Institute of Marine Research and Dr Jesús Lamas (University of Santiago de Compostela) for their help with the histological processing.

\section{References}

Abu-Shanab A \& Quigley EMM 2010 The role of the gut microbiota in nonalcoholic fatty liver disease. Nature Reviews. Gastroenterology \& Hepatology 7 691-701. (doi:10.1038/nrgastro.2010.172)

Aigner E, Theurl I, Theurl M, Lederer D, Haufe H, Dietze O, Strasser M, Datz C \& Weiss G 2008 Pathways underlying iron accumulation in human nonalcoholic fatty liver disease. American Journal of Clinical Nutrition 87 1374-1383.

Anezaki Y, Ohshima S, Ishii H, Kinoshita N, Dohmen T, Kataoka E, Sato W, Iizuka M, Goto T, Sasaki J et al. 2009 Sex difference in the liver of hepatocyte-specific Pten-deficient mice: a model of nonalcoholic steatohepatitis. Hepatology Research 39 609-618. (doi:10.1111/j.1872034X.2009.00494.x)

Armutcu F, Akyol S, Ucar F, Erdogan S \& Akyol O 2013 Markers in nonalcoholic steatohepatitis. Advances in Clinical Chemistry 61 67-125. (doi:10.1016/B978-0-12-407680-8.00004-X)

Asaoka Y, Terai S, Sakaida I \& Nishina H 2013 The expanding role of fish models in understanding non-alcoholic fatty liver disease. Disease Models \& Mechanisms 6 905-914. (doi:10.1242/dmm.011981)

Bessesen DH 2008 Update on obesity. Journal of Clinical Endocrinology and Metabolism 93 2027-2034. (doi:10.1210/jc.2008-0520)

Bindea G, Mlecnik B, Hackl H, Charoentong P, Tosolini M, Kirilovsky A, Fridman W-H, Pagès F, Trajanoski Z \& Galon J 2009 ClueGO: a cytoscape plug-in to decipher functionally grouped gene ontology and pathway annotation networks. Bioinformatics 25 1091-1093. (doi:10.1093/bioinformatics/btp101)

Chu C-Y, Chen C-F, Rajendran RS, Shen C-N, Chen T-H, Yen C-C, Chuang C-K, Lin D-S \& Hsiao C-D 2012 Overexpression of Akt1 enhances adipogenesis and leads to lipoma formation in zebrafish. PLOS ONE 7 e36474. (doi:10.1371/journal.pone.0036474)

Cinaroglu A, Gao C, Imrie D \& Sadler KC 2011 Activating transcription factor 6 plays protective and pathological roles in steatosis due to endoplasmic reticulum stress in zebrafish. Hepatology 54 495-508. (doi:10.1002/hep.24396)
Conesa A, Götz S, García-Gómez JM, Terol J, Talón M \& Robles M 2005 Blast2GO: a universal tool for annotation, visualization and analysis in functional genomics research. Bioinformatics 21 3674-3676. (doi:10.1093/bioinformatics/bti610)

Duan XY, Zhang L, Fan JG \& Qiao L 2013 NAFLD leads to liver cancer: do we have sufficient evidence? Cancer Letters 345 230-234. (doi:10.1016/ j.canlet.2013.07.033)

Fèvre-Montange M, Champier J, Durand A, Wierinckx A, Honnorat J, Guyotat J \& Jouvet A 2009 Microarray gene expression profiling in meningiomas: differential expression according to grade or histopathological subtype. International Journal of Oncology 35 1395-1407. (doi:10.3892/ijo_00000457)

Fouts DE, Torralba M, Nelson KE, Brenner DA \& Schnabl B 2012 Bacterial translocation and changes in the intestinal microbiome in mouse models of liver disease. Journal of Hepatology 56 1283-1292. (doi:10.1016/j.jhep.2012.01.019)

Garruti G, Wang HH, Bonfrate L, de Bari O, Wang DQ-H \& Portincasa P 2012 A pleiotropic role for the orphan nuclear receptor small heterodimer partner in lipid homeostasis and metabolic pathways. Journal of Lipids 2012 304292. (doi:10.1155/2012/304292)

Henao-Mejia J, Elinav E, Jin C, Hao L, Mehal WZ, Strowig T, Thaiss CA, Kau AL, Eisenbarth SC, Jurczak MJ et al. 2013 Inflammasome-mediated dysbiosis regulates progression of NAFLD and obesity. Nature $\mathbf{4 8 2}$ 179-185. (doi:10.1038/nature10809)

Huang DW, Sherman BT \& Lempicki RA 2009a Bioinformatics enrichment tools: paths toward the comprehensive functional analysis of large gene lists. Nucleic Acids Research 37 1-13. (doi:10.1093/nar/gkn923)

Huang DW, Sherman BT \& Lempicki RA 2009b Systematic and integrative analysis of large gene lists using DAVID bioinformatics resources. Nature Protocols 4 44-57. (doi:10.1038/nprot.2008.211)

Jones KS, Alimov AP, Rilo HL, Jandacek RJ, Woollett LA \& Penberthy WT 2008 A high throughput live transparent animal bioassay to identify non-toxic small molecules or genes that regulate vertebrate fat metabolism for obesity drug development. Nutrition and Metabolism 5 23. (doi:10.1186/1743-7075-5-23)

Kitamura A, Takahashi K, Okajima A \& Kitamura N 1994 Induction of the human gene for $\mathrm{p} 44$, a hepatitis-C-associated microtubular aggregate protein, by interferon $-\alpha / \beta$. European Journal of Biochemistry 224 877-883. (doi:10.1111/j.1432-1033.1994.00877.x)

Kowdley KV, Kowdley KV, Belt P, Belt P, Wilson LA, Wilson LA, Yeh MM, Yeh MM, Neuschwander-Tetri BA, Neuschwander-Tetri BA et al. 2012 Serum ferritin is an independent predictor of histologic severity and advanced fibrosis in patients with nonalcoholic fatty liver disease. Hepatology 55 77-85. (doi:10.1002/hep.24706)

Krücken J, Schroetel RMU, Müller IU, Saïdani N, Marinovski P, Benten WPM, Stamm O \& Wunderlich F 2004 Comparative analysis of the human gimap gene cluster encoding a novel GTPase family. Gene 341 291-304. (doi:10.1016/j.gene.2004.07.005)

Krzywinski M, Schein J, Birol I, Connors J, Gascoyne R, Horsman D, Jones SJ \& Marra MA 2009 Circos: an information aesthetic for comparative genomics. Genome Research 19 1639-1645. (doi:10.1101/gr. 092759.109)

Li S, Zhang H-Y, Hu CC, Lawrence F, Gallagher KE, Surapaneni A, Estrem ST, Calley JN, Varga G, Dow ER et al. 2008 Assessment of diet-induced obese rats as an obesity model by comparative functional genomics. Obesity $\mathbf{1 6}$ 811-818. (doi:10.1038/oby.2007.116)

MacLaren RE, Cui W, Lu H, Simard S \& Cianflone K 2010 Association of adipocyte genes with ASP expression: a microarray analysis of subcutaneous and omental adipose tissue in morbidly obese subjects. BMC Medical Genomics 3 3. (doi:10.1186/1755-8794-3-3)

Mehlem A, Hagberg CE, Muhl L, Eriksson U \& Falkevall A 2013 Imaging of neutral lipids by oil red $\mathrm{O}$ for analyzing the metabolic status in health and disease. Nature Protocols 8 1149-1154. (doi:10.1038/nprot. 2013.055)

Metwali A, Blum AM, Elliott DE, Setiawan T \& Weinstock JV 2004 Cutting edge: hemokinin has substance P-like function and expression in 
inflammation. Journal of Immunology 172 6528-6532. (doi:10.4049/ jimmunol.172.11.6528)

Michalová V, Murray BW, Sültmann H \& Klein J 2000 A contig map of the Mhc class I genomic region in the zebrafish reveals ancient synteny. Journal of Immunology 164 5296-5305. (doi:10.4049/jimmunol.164.10.5296)

Miura K, Yang L, van Rooijen N, Brenner DA, Ohnishi H \& Seki E 2013 Toll-like receptor 2 and palmitic acid cooperatively contribute to the development of nonalcoholic steatohepatitis through inflammasome activation in mice. Hepatology 57 577-589. (doi:10.1002/hep.26081)

Nadler ST, Stoehr JP, Schueler KL, Tanimoto G, Yandell BS \& Attie AD 2000 The expression of adipogenic genes is decreased in obesity and diabetes mellitus. PNAS 97 11371-11376. (doi:10.1073/pnas.97.21.11371)

Nusslein-Volhard C \& Dahm R 2002 Zebrafish. New York, NY: Oxford University Press.

Oka T, Nishimura Y, Zang L, Hirano M, Shimada Y, Wang Z, Umemoto N, Kuroyanagi J, Nishimura N \& Tanaka T 2010 Diet-induced obesity in zebrafish shares common pathophysiological pathways with mammalian obesity. BMC Physiology 1021 . (doi:10.1186/1472-6793-10-21)

Page NM 2004 Hemokinins and endokinins. Cellular and Molecular Life Sciences 61 1652-1663. (doi:10.1007/s00018-004-4035-x)

Page NM 2006 Characterization of the gene structures, precursor processing and pharmacology of the endokinin peptides. Vascular Pharmacology 45 200-208. (doi:10.1016/j.vph.2005.08.028)

Pelz S, Stock P, Brückner S \& Christ B 2012 A methionine-choline-de. Experimental Cell Research 318 276-287. (doi:10.1016/j.yexcr.2011.11.005)

Pessayre D 2007 Role of mitochondria in non-alcoholic fatty liver disease. Journal of Gastroenterology and Hepatology 22 S20-S27. (doi:10.1111/ j.1440-1746.2006.04640.x)

Pfaffl MW 2001 A new mathematical model for relative quantification in real-time RT-PCR. Nucleic Acids Research 29 e45. (doi:10.1093/nar/ 29.9.e45)

Poussin C, Hall D, Minehira K, Galzin A-M, Tarussio D \& Thorens B 2008 Different transcriptional control of metabolism and extracellular matrix in visceral and subcutaneous fat of obese and rimonabant treated mice. PLoS ONE 3 e3385. (doi:10.1371/journal.pone.0003385)

Radonjic M, de Haan JR, van Erk MJ, van Dijk KW, van den Berg SAA, de Groot PJ, Müller M \& van Ommen B 2009 Genome-wide mRNA expression analysis of hepatic adaptation to high-fat diets reveals switch from an inflammatory to steatotic transcriptional program. PLoS ONE 4 e6646. (doi:10.1371/journal.pone.0006646)

Roh YS \& Seki E 2013 Toll-like receptors in alcoholic liver disease, non-alcoholic steatohepatitis and carcinogenesis. Journal of Gastroenterology and Hepatology 28 38-42. (doi:10.1111/jgh.12019)

Rozen S \& Skaletsky H 2000 Primer3 on the WWW for general users and for biologist programmers. Methods in Molecular Biology (Clifton, N.J.) 132 365-386.

Sadler KC, Amsterdam A, Soroka C, Boyer J \& Hopkins N 2005 A genetic screen in zebrafish identifies the mutants vps18, nf2 and foie gras as models of liver disease. Development (Cambridge, England) 132 3561-3572. (doi:10.1242/dev.01918)

Sahar S \& Sassone-Corsi P 2012 Regulation of metabolism: the circadian clock dictates the time. Trends in Endocrinology and Metabolism 23 1-8. (doi:10.1016/j.tem.2011.10.005)

Scheja L, Heese B, Zitzer H, Michael MD, Siesky AM, Pospisil H, Beisiegel U \& Seedorf K 2008 Acute-phase serum amyloid A as a marker of insulin resistance in mice. Experimental Diabetes Research 2008 1-11. (doi:10.1155/2008/230837)

Schlegel A \& Stainier DYR 2007 Lessons from "Lower" organisms: what worms, flies, and zebrafish can teach us about human energy metabolism. PLoS Genetics 3 e199. (doi:10.1371/journal.pgen.0030199)
Sepulcre MP, Alcaraz-Perez F, Lopez-Munoz A, Roca FJ, Meseguer J, Cayuela ML \& Mulero V 2009 Evolution of lipopolysaccharide (LPS) recognition and signaling: fish TLR4 does not recognize LPS and negatively regulates NF-KB activation. Journal of Immunology 182 1836-1845. (doi:10.4049/jimmunol.0801755)

Shieh Y-S, Chang Y-S, Hong J-R, Chen L-J, Jou L-K, Hsu C-C \& Her GM 2010 Increase of hepatic fat accumulation by liver specific expression of hepatitis B virus X protein in zebrafish. Biochimica et Biophysica Acta 1801 721-730. (doi:10.1016/j.bbalip.2010.04.008)

Shirakami Y, Lee S-A, Clugston RD \& Blaner WS 2012 Hepatic metabolism of retinoids and disease associations. Biochimica et Biophysica Acta 1821 124-136. (doi:10.1016/j.bbalip.2011.06.023)

Smalling RL, Delker DA, Zhang Y, Nieto N, McGuiness MS, Liu S, Friedman SL, Hagedorn CH \& Wang L 2013 Genome-wide transcriptome analysis identifies novel gene signatures implicated in human chronic liver disease. American Journal of Physiology. Gastrointestinal and Liver Physiology 305 G364-G374. (doi:10.1152/ajpgi.00077.2013)

Subramanian A, Tamayo P, Mootha VK, Mukherjee S, Ebert BL, Gillette MA, Paulovich A, Pomeroy SL, Golub TR, Lander ES et al. 2005 Gene set enrichment analysis: a knowledge-based approach for interpreting genome-wide expression profiles. PNAS 102 15545-15550. (doi:10.1073/pnas.0506580102)

Sullivan C, Charette J, Catchen J, Lage CR, Giasson G, Postlethwait JH, Millard PJ \& Kim CH 2009 The gene history of zebrafish tlr4a and tlr4b is predictive of their divergent functions. Journal of Immunology $\mathbf{1 8 3}$ 5896-5908. (doi:10.4049/jimmunol.0803285)

Tahara Y, Otsuka M, Fuse Y, Hirao A \& Shibata S 2011 Refeeding after fasting elicits insulin-dependent regulation of Per2 and Rev-erb with shifts in the liver clock. Journal of Biological Rhythms 26 230-240. (doi:10.1177/0748730411405958)

Tainaka T, Shimada Y, Kuroyanagi J, Zang L, Oka T, Nishimura Y, Nishimura N \& Tanaka T 2011 Transcriptome analysis of anti-fatty liver action by Campari tomato using a zebrafish diet-induced obesity model. Nutrition and Metabolism 8 88. (doi:10.1186/1743-7075-8-88)

Tsolmongyn B, Koide N, Odkhuu E, Haque A, Naiki Y, Komatsu T, Yoshida T \& Yokochi T 2013 Lipopolysaccharide prevents valproic acid-induced apoptosis via activation of nuclear factor- $\kappa \mathrm{B}$ and inhibition of $\mathrm{p} 53$ activation. Cellular Immunology 282 100-105. (doi:10.1016/j.cellimm. 2013.04.011)

Vadasz Z, Kessler O, Akiri G, Gengrinovitch S, Kagan HM, Baruch Y, Izhak OB \& Neufeld G 2005 Abnormal deposition of collagen around hepatocytes in Wilson's disease is associated with hepatocyte specific expression of lysyl oxidase and lysyl oxidase like protein-2. Journal of Hepatology 43 499-507. (doi:10.1016/j.jhep.2005.02.052)

Westerfield M 2000 The Zebrafish Book: A Guide for the Laboratory Use of Zebrafish (Danio Rerio). Eugene: Univ. of Oregon Press.

Yamashita-Sugahara Y, Tokuzawa Y, Nakachi Y, Kanesaki-Yatsuka Y, Matsumoto M, Mizuno Y \& Okazaki Y 2013 Fam57b (family with sequence similarity 57 , member $\mathrm{B}$ ), a novel peroxisome proliferatoractivated receptor target gene that regulates adipogenesis through ceramide synthesis. Journal of Biological Chemistry $2884522-4537$. (doi:10.1074/jbc.M112.440792)

Yu J, Shen J, Sun TT, Zhang X \& Wong N 2013 Obesity, insulin resistance, NASH and hepatocellular carcinoma. Seminars in Cancer Biology 23 483-491. (doi:10.1016/j.semcancer.2013.07.003)

Zani F, Breasson L, Becattini B, Vukolic A, Montani J-P, Albrecht U, Provenzani A, Ripperger JA \& Solinas G 2013 PER2 promotes glucose storage to liver glycogen during feeding and acute fasting by inducing Gys2 PTG and G. Molecular Metabolism 2 1-14.

Received in final form 28 October 2014

Accepted 4 November 2014

Accepted Preprint published online 4 November 2014 http://joe.endocrinology-journals.org DOI: 10.1530/JOE-14-0398
() 2015 Society for Endocrinology Printed in Great Britain 